\title{
Experimental Demonstration of Optical Multicast Packet Transmissions in Optical Packet/Circuit Integrated Networks
}

\author{
Yusuke Hirota $^{1 *}$, Sugang Xu $\mathbf{X}^{1}$, Masaki Shiraiwa ${ }^{1}$, Yoshinari Awaji ${ }^{1}$, Massimo Tornatore ${ }^{2,3}$, \\ Biswanath Mukherjee $^{2}$, Hideaki Furukawa ${ }^{1}$, and Naoya Wada ${ }^{1}$ \\ ${ }^{I}$ National Institution of Information and Communications Technology, Japan \\ ${ }^{2}$ University of California, Davis, USA \\ ${ }^{3}$ Politecnico di Milano, Italy \\ *hirota.yusuke@nict.go.jp
}

\begin{abstract}
We develop an SDN-based control for optical-multicast packet transmission and experimentally demonstrate multicast functionality by validating it using an application-layer network service for efficient content duplication in Optical Packet/Circuit Integrated (OPCI) network. (C) 2020 The Authors
\end{abstract}

OPCI codes: 060.4255 Networks, multicast; 060.4259 Networks, packet-switched

\section{Introduction}

To support effective implementation of content delivery or data backup, a large amount of data must be continuously exchanged among all the data centers where data is replicated, and content duplication must be conducted efficiently and with low processing load and latency. Multicast is an efficient transmission technique to transmit data to multiple destinations. Electrical multicast using electrical router/switch such as IP multicast is well studied. Ref. [1] proposes dynamic multicast traffic engineering for software defined networks. Yet, in case of electrical multicast, router/switch must duplicate a huge number of packets through electrical processing, that is energy-hungry and adds non-negligible latency. Instead, optical multicast is suitable for multicast transmission of large-size data, because optical signals can be easily broadcasted to many output ports in a switching node without additional latency and with minimal additional energy consumption. Unfortunately, optical multicast has still several issues to be addressed, as: i) a stricter wavelength continuity constraint along all multicast tree must be satisfied [2], and ii) current commercial transponders (e.g., current $10 \mathrm{G}$ transmitters) cannot execute parallel handshake processes, which are needed before multicast transmissions to multiple $10 \mathrm{G}$ receivers simultaneously. Both these issues can be resolved if, instead of traditional optical circuit multicast, we consider optical packet multicast. In this paper, we first develop an SDN-based control scheme for optical multicast packet transmissions to realize content duplication in an Optical Packet/Circuit Integrated (OPCI) network (Fig. 1). We then experimentally demonstrate optical multicast transmission and we test the effectiveness of optical multicast experimenting the transmission of an application-layer streaming service.

\section{SDN-based Optical Multicast Transmission for Efficient Content Duplication}

In case of multiple failures, network connectivity might be lost even if some protection schemes are adopted. Ref. [3] and [4] have studied how to ensure content connectivity during survivable virtual network mapping on a physical optical network in case of single and double failure, respectively. Still, it remains to be understood how to distribute content replicas to multiple nodes to enable content connectivity. There are mainly three types of transmissions for distributing data to multiple destinations: $i$ ) serial unicast transmission, ii) parallel unicast transmission, and iii) multicast transmission. In serial unicast transmission, a sender simply transmits data to multiple destinations one by one,

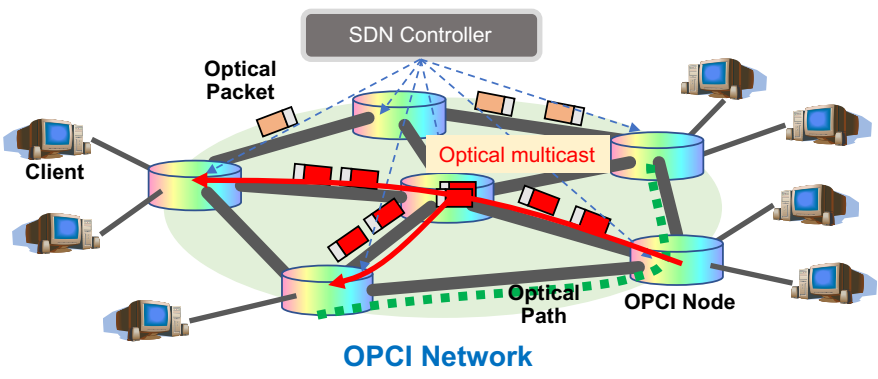

Fig. 1. SDN-based OPCI control mechanism.

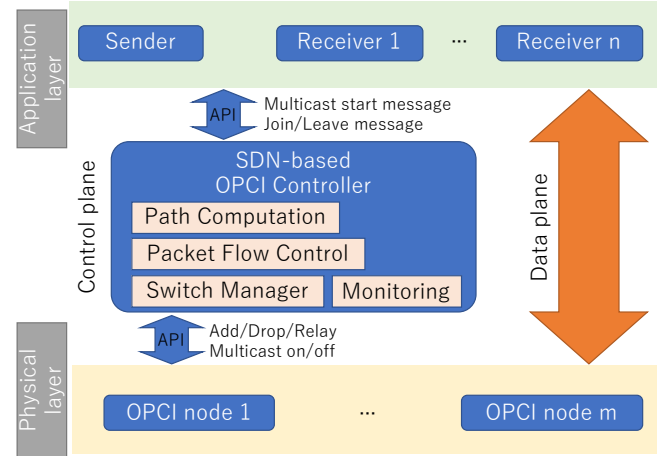

Fig. 2. OPCI Network and Optical Multicast. 
hence taking long time to complete data replication. In parallel unicast transmission, a sender transmits data to multiple destinations simultaneously. The required transmission time is shortened, however large amount of capacity is necessary, and this might cause network congestion or connection blocking. In multicast transmission, a sender sends data only once, then some intermediate network nodes duplicate data stream to reach multiple destination nodes. This is the best approach in terms of latency and network load. Multicast has been investigated at different network layers in recent years., SDN-based multicast transmission is studied in [1]. Through an SDN-based approach, it is easy to control multicast transmissions, particularly join/leave process of destination nodes. Optical multicast has been shown to be suitable for large data transfer to multiple destinations, e.g., as shown for the optical multicast scheme using an active vertical coupler-based optical switch matrix proposed in [5]. However, there are no experimental studies showing how optical packet multicast be used to can support existing network applications over real optical devices. In our work, we developed API for configuration of optical multicast packet transmission. The high-level system model is shown in Fig. 2. When the SDN-based OPCI controller receives multicast-related messages from a sender or receivers, the OPCI controller executes adequate control procedures such as multicast route calculation, validation of switch configuration, and sends switch configuration messages to the related OPCI nodes. After switch configuration, optical multicast transmission or unicast transmission in data plane starts. When sender stops data transmission or a receiver sends leave message to OPCI controller, OPCI controller reconfigures OPCI nodes to stop multicast or unicast transmissions.

\section{Experimental Demonstration of Optical Packet Multicast in Ring based OPCI Network}

To verify the functionality of optical level packet multicast, we developed API-based optical multicast procedure and demonstrated multicast transmission in ring-based OPCI network. Figure 4 shows the experimental setup. We use one OPCI controller, four OPCI nodes, 4 layer- 2 switches, 4 router testers, and three computers. Note that detailed information of OPCI node is reported in Ref. [6]. We first demonstrated a background traffic scenario. The demonstration is shown in Fig. 4. Figs. 4a, $4 b$, and $4 c$ displays the number of transmitting/receiving packets router testers, Fig. 4d shows the command line interface of OPCI controller, and Figs. $4 \mathrm{c}$ and $4 \mathrm{f}$ is the real-time measured results at the output fiber of OPCI-1 by a spectrum analyzer and an oscilloscope, separately. RT-1 firstly sent ARP requests to RT-2 and RT-3 and then sent unicast packets to RT-2 and RT-3 separately in order to check network connectivity of RT-1-RT-2 and RT1-RT-3. Next, RT-1 sent multicast packets into the OPCI network. Only RT-2 received these multicast packets from RT-1 through L2-SW-1, OPCI-1, OPCI-2, and L2-SW-2 when OPCI controller did not enable multicast function at OPCI-2. In 45 seconds after starting multicast packets, OPCI controller sends multicast-on message to OPCI-2 and "drop" message to OPCI-3 in order for RT-3 to receive these multicast packets. Then optical signal of these packets was duplicated at OPCI-2 and one of them was dropped and transmitted to PC-a2, and the other was transmitted to OPCI-3. From Fig. 4, we confirmed that optical packets are successfully transmitted to two destinations using optical multicast function at OPCI-2. We also verified the effectiveness of the optical level multicast packet transmissions for end-to-end application services. As a sample layer-7 application, we adopted video streaming. PC-al sent streaming data as optical multicast packets, whose destination address was one of IP multicast addresses, using VLC [7] server. Firstly, PC-a2 sent join message to OPCI controller and then OPCI controller configured OPCI-1 and OPCI-2 to transmit the streaming data to PC-a2. After a few seconds, $\mathrm{PC}$-a2 received the streaming data and displayed the video. Next, $\mathrm{PC}$-a 3 also sent join message

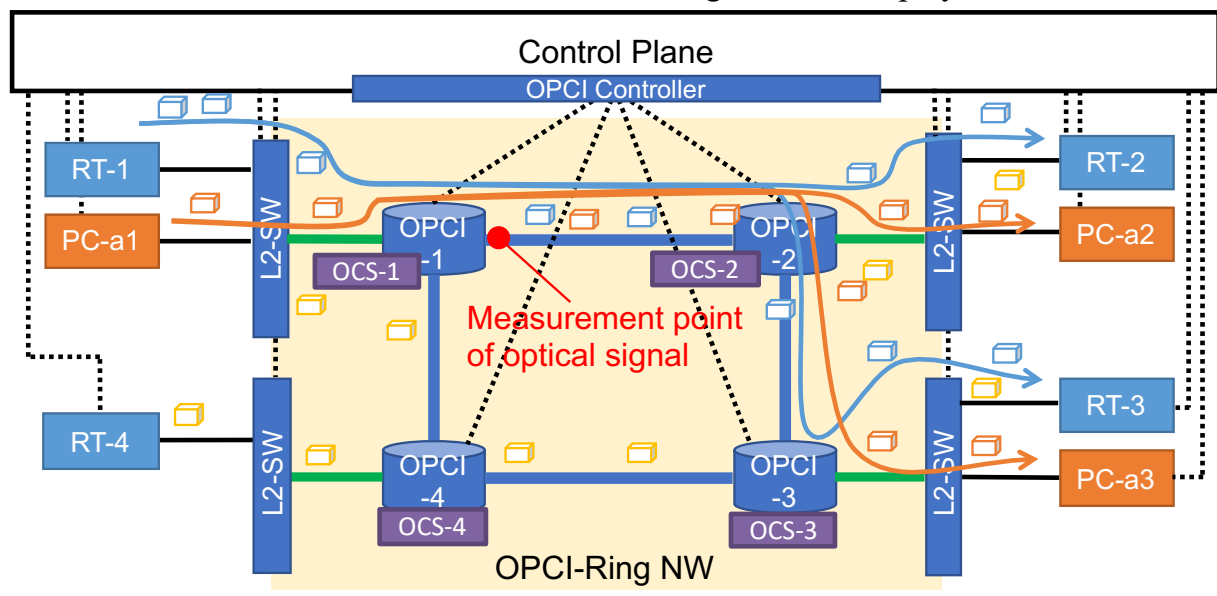

Fig. 3. Experimental setup of OPCI network.

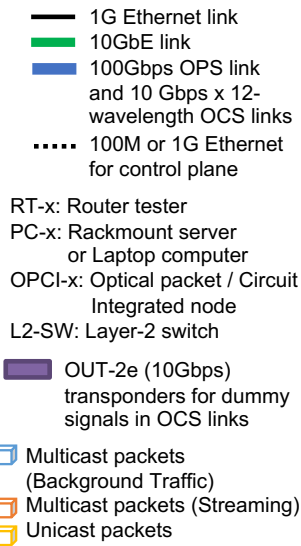


to OPCI controller as PC-a2 did. OPCI controller sent "multicast-on" message to OPCI-2 and "drop" message to OPCI-3. Then, PC-a3 also received the same packets and display the same video simultaneously. Figure 5 shows the streaming demonstrations. Fig. 5a shows the VLC media player for streaming multicast packets. Fig. 5b is CLI window to execute VLC at PC-a1. Fig. 5c is CLI window of OPCI controller. Fig. 5d displays the captured packets by tcpdump. Figs. $5 \mathrm{e}, 5 \mathrm{f}$, and $5 \mathrm{~g}$ show the windows of VLC player, CLI to use API for multicasting, and the captured packets at PC-a2, separately. Figs. 5h, 5i, and 5j show the same windows at PC-a3. Firstly, PC-a1 start to send streaming video. Then, PC-a2 and PC-a3 sent "join" message using CLI-based API and also execute VLC receiver. When OPCI controller receives these "join" messages, OPCI controller configures related OPCI nodes to send optical multicast packets. We confirm the application-level operation using VLC and we also confirmed the two receivers received the same packets from three windows of tcpdump at PC-a1, PC-a2, and PC-a3. We successfully confirmed that the sending packets displayed in Fig. 5 in terms of not only packet capture but also application service.

\section{Acknowledgement}

This work is partially supported by US-Japan JUNO2 project: NSF Grant no. 1818972. The authors wish to thank Mr. Weiping Ren for his technical support.

\section{References}

[1] J-J. Kuo, et al., "Dynamic Multicast Traffic Engineering with Efficient Rerouting for SoftwareDefined Networks," INFOCOM, July 2019.

[2] Y. Hirota, et al., "Multicast design method using multiple shared-trees in optical WDM networks," IEICE E95-B(2), 370-381, Feb. 2012.

[3] M. F. Habib, et al., "Fault-tolerant virtual network mapping to provide content connectivity in optical networks," OFC, Mar. 2013.

[4] A. Hmaity, et al., "Survivable virtual network mapping to provide content connectivity against double-link failures," DRCN, Mar. 2016.

[5] S. Yu, et al., "Lossless optical packet multicast using active vertical coupler based optical crosspoint switch matrix," JLT 23(10), 2984-2992, Oct. 2005.

[6] H. Furukawa, et al., "Development of optical

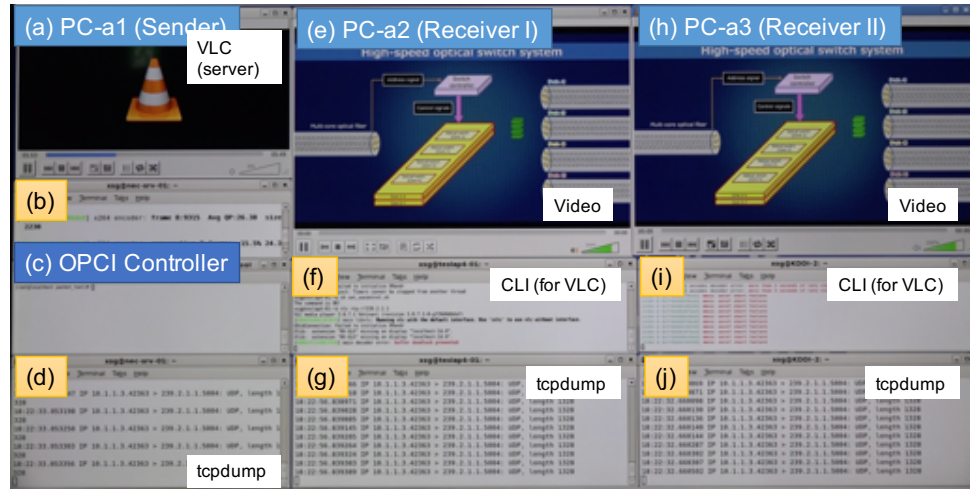
packet and circuit integrated ring network testbed," Opt. Express 19(26), B242-B250 (2011).

[7] VLC media player, https://www.videolan.org/index.html

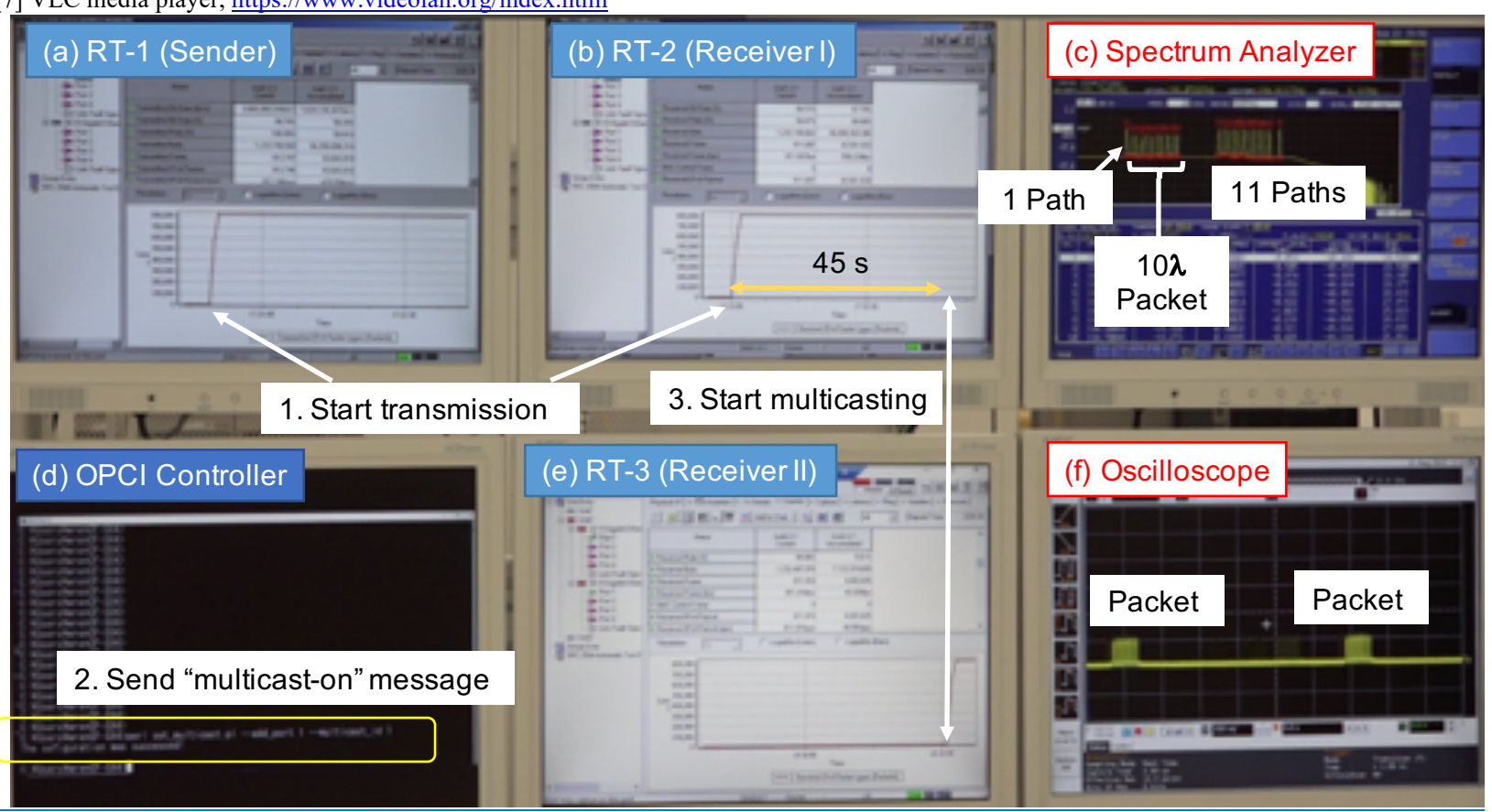

Fig. 4. Demonstration. (a) \# of transmitted packets of RT-1, (b) \# of received packets of RT-2, (c) spectrum analyzer, (d) command line terminal of OPCI controller, (e) \# of received packets of RT-3, (f) Oscilloscope. 